Tropical Journal of Pharmaceutical Research January 2016; 15 (1): 55-64

ISSN: $1596-5996$ (print); 1596-9827 (electronic)

(C) Pharmacotherapy Group, Faculty of Pharmacy, University of Benin, Benin City, 300001 Nigeria.

All rights reserved.

Available online at http://www.tjpr.org

Original Research Article

http://dx.doi.org/10.4314/tjpr.v15i1.8

\title{
Effects of Triptergium Glycosides on Expressions of MCP- 1 and CTGF in Rats with Early Diabetic Nephropathy
}

\author{
Ji-Qiang Zhang, Yan Zhang, Xiao-Li Yin, Ping Yang, Hai-Feng Zhang, Ya-Ling \\ Guo and Wei-Dong Chen* \\ Department of Nephrology, No. 1 Affiliated Hospital of Bengbu Medical College, Bengbu 233004, PR China
}

${ }^{*}$ For correspondence: Email: wdchen_hbmc@126.com

Received: 4 August 2015

Revised accepted: 12 December 2015

\begin{abstract}
Purpose: To investigate the effects of triptergium glycosides (TG) on expressions of monocyte chemoattractant protein-1 (MCP-1) and connective tissue growth factor (CTGF) in early diabetic nephropathy $(D N)$ in rats, and explore its mechanism of renal protection.

Methods: Thirty-two rats were divided into 4 groups: normal control (NC), DN, and DN-treated with TG (5 and $10 \mathrm{mg} / \mathrm{kg} / \mathrm{d}$ ) groups. After 8 weeks, body weight, blood glucose (BG), albumin (ALB), blood urea nitrogen (BUN), creatinine (SCr) and $24 \mathrm{~h}$ urinary total protein (UTP) of rats were determined. Additionally, expressions of CTGF, MCP-1 and ED-1 were detected by immunohistochemistry assay, while mRNA and protein expressions of CTGF and MCP-1 in kidney tissues were evaluated using reverse transcription-polymerase chain reaction (RT-PCR) and western blot technique.

Results: BG, ALB, SCr, BUN and UTP in DN group were significantly increased $(p<0.01)$, compared with NC group. Compared with DN group, ALB (28.90 and $31.49 \mathrm{vs} 23.13 \mathrm{~g} / \mathrm{L})$ and UTP (21.87 and $18.91 \mathrm{vs} 37.19 \mathrm{mg} / 24 \mathrm{~h})$ were significantly changed in TG groups $(p<0.05)$. ED-1 positive cells were significantly increased in $D N$ group $(p<0.01)$, compared with NC group, whereas treatment with TG significantly reversed the increase (1.67 and 1.41 vs 2.73 in glomeruli, 9.86 and 9.49 VS 13.18 in glomerular interstitial, $p<0.01$ ). Proteins and mRNA expressions of CTGF and MCP-1 significantly increased $(p<0.01)$ in $D N$ group, compared with NC group, while their expressions in TG groups were reversed.

Conclusion: TG ameliorates renal injury in diabetic rats via decreasing MCP-1 and CTGF expressions and reducing macrophage activation.
\end{abstract}

Keywords: Diabetic nephropathy, Triptergium glycosides, Connective tissue growth factor, Monocyte chemoattractant protein-1

Tropical Journal of Pharmaceutical Research is indexed by Science Citation Index (SciSearch), Scopus, International Pharmaceutical Abstract, Chemical Abstracts, Embase, Index Copernicus, EBSCO, African Index Medicus, JournalSeek, Journal Citation Reports/Science Edition, Directory of Open Access Journals (DOAJ), African Journal Online, Bioline International, Open-J-Gate and Pharmacy Abstracts

\section{INTRODUCTION}

Increasing clinical and animal studies have indicated that activation of the immune response and inflammatory mechanisms are important in the pathogenesis of diabetic nephropathy (DN), which is one of the chronic complications of diabetes [1]. It's reported that chemokines, such as monocyte chemoattractant protein-1 (MCP-1) and connective tissue growth factor (CTGF), play important roles in the recruitment and activation of these immunocytes. Specifically, MCP-1 can cause extensive migration and infiltration of monocytes/macrophages in kidney actively involved in DN [2]. CTGF, a downstream effector of transforming growth factor- $\beta 1$ (TGF- $\beta 1$ ), stimulates mesangial cell proliferation and extracellular matrix (ECM) production and also 
promotes the development of renal fibrosis, leading to renal pathological changes associated with DN [3].

Triptergium glycoside (TG) is commonly used to treat DN based on its anti-inflammatory and immunosuppressive effects. However, the specific mechanism of TG's renal protection is still not entirely clear. Therefore, the present study was designed to investigate the effect of TG on expressions of MCP-1 and CTGF in early $\mathrm{DN}$ rats and to explore its mechanism of renal protection.

\section{EXPERIMENTAL}

\section{Materials and chemicals}

TG was purchased from Fudan Forward Co. (Shanghai, China). Streptozotocin (STZ) was purchased from Sigma Co. (USA). The following reagents were purchased from Aobosen Co. (Beijing, China): rabbit anti-rat CTGF and MCP-1 primary antibodies, mouse anti-rat ED-1 monoclonal antibody, goat anti-rabbit IgG antibody, goat anti-mouse IgG antibody and DAB horseradish peroxidase color development kit. Trizol reagent was purchased from Invitrogen Co. (USA), and RT-PCR kit was purchased from Fermentas Co. (USA). CTGF, MCP-1 and $\beta$-actin primers were synthesized by Sangon Biotech. Co., Shanghai, China. All other reagents used were of analytic grade.

\section{Animals}

Male Sprague Dawley (SD) rats (8-weeks old, weighing 180 - $200 \mathrm{~g}$ ) were purchased from Experimental Animal Center of Bengbu Medical College (Anhui, China). All the animal protocols were established according to the generally accepted international rules strictly, and approved by the Animal Care and Use Committee of our College.

\section{Experiment design}

Thirty-two male SD rats were used in the present study. Diabetic rats were constructed by feeding a high-fat and high-sugar diet with $10 \%$ lard, 20 $\%$ sucrose and $3 \%$ cholesterol for 4 weeks, and given a single intraperitoneal injection (ip) of 55 $\mathrm{mg} / \mathrm{kg} \mathrm{STZ}$ in sodium citrate. Meanwhile, the rats in negative control group (NC group, $\mathrm{n}=8$ ) were fed a normal diet, and injected an equal volume of citrate-citrate sodium buffer alone (ip). Diabetes was confirmed if the blood glucose (BG) value on the third day exceeded 16.7 $\mathrm{mmol} / \mathrm{L}$ after STZ injection. Moreover, DN was confirmed if the $24 \mathrm{~h}$ urinary total protein (UTP) values exceeded $30 \mathrm{mg}$, and the amount of $24 \mathrm{~h}$ urine was increased by $50 \%$, compared with before induction.

After the DN rats were rendered diabetic, they were randomly divided into 3 groups, including DN group, and TG treatment groups $[5 \mathrm{mg} / \mathrm{kg} / \mathrm{d}$ (TG1) and $10 \mathrm{mg} / \mathrm{kg} / \mathrm{d}(\mathrm{TG} 2)$ ] ( $\mathrm{n}=8)$. TG (5 and $10 \mathrm{mg} / \mathrm{kg} / \mathrm{d}$ ) was administered orally (ig) once a day for 8 weeks. Rats in the NC and DN groups received an equivalent volume of physiological saline per day (ig). During the experimental period, antidiabetic drugs were not applied. Body weight was measured weekly, and $24 \mathrm{~h}$ urine was collected after induction and analyzed at the end of the study. All the animals were sacrificed after hemospasia from the heart, and then blood samples were centrifuged to obtain serum. Serum samples were stored at $-20{ }^{\circ} \mathrm{C}$ until analysis. The kidneys (right and left) were dissected out and fixed in $10 \%$ formalin for histopathological analysis or fixed in $3.75 \%$ pentanediol for transmission electron microscopy (TEM) assay. The rest of the kidney tissues were rapidly frozen with liquid nitrogen and stored at $80^{\circ} \mathrm{C}$.

\section{Blood sampling and biochemical analysis}

By using the automatic biochemistry analyzer, blood and urine samples of rats were collected to determine the albumin (ALB), blood urea nitrogen (BUN), creatinine (SCr), UTP and other indicators. BG was measured using blood glucose monitor (Roche, Switzerland).

\section{Electron microscopy (EM)}

Tissue fragments $(1 \mathrm{~mm} 3)$ were randomly taken from the kidney tissue and fixed in $3.75 \%$ pentanediol at $4{ }^{\circ} \mathrm{C}$ for more than $4 \mathrm{~h}$, washed five or six times with $0.1 \mathrm{M}$ PBS, dehydrated in ethanol, and embedded in paraffin. For electron microscopy, blocks were trimmed and sections were cut at 80 - $90 \mathrm{~nm}$, mounted on copper grids, aluminum citrate stained, and viewed under the electron microscope (JEOL).

\section{Histopathological examination}

The tissue sections were stained by hematoxylin and eosin (H\&E) using standard histology procedures. Immunohistochemical staining was performed using SABC kit according to the manufacturer's instructions of commercial kits. The HPIAS-1000 high-resolution color graphic report of the pathology analysis system was used to analyze the average of the integral optical density (IDP). In each section, the number of ED1 positive cells of 10 randomly selected 
glomerular fields and 10 non-overlapping renal tubule-interstitial fields under high power magnification $(400 x)$ were counted.

\section{Western blot assay}

Western blot assay was performed to determine the expressions of MCP-1 and CTGF in kidney tissues. Kidney tissues were homogenized in RIPA lysis buffer supplemented with protease inhibitors and phosphatase inhibitors, using a 10 $\% \mathrm{w} / \mathrm{v}$ ratio. Homogenates were then centrifuged for $15 \mathrm{~min}$ at $11,000 \mathrm{~g}$ and the supernatants were stored at $-80{ }^{\circ} \mathrm{C}$. Total proteins were extracted, and the concentration was determined using a $\mathrm{BCA}$ reagent kits. Thereafter, the proteins were separated by electrophoresis on sodium dodecyl sulfate/polyacrylamide gel (SDS-PAGE). Following transfer onto polyvinylidene fluoride (PVDF) membranes and blocking, the membranes were incubated overnight at $4{ }^{\circ} \mathrm{C}$ with the primary antibodies of MCP-1, CTGF and $\beta$-actin (1: 100). Following several washes in TBST, the PVDF membranes were subsequently incubated with horseradish peroxidaseconjugated secondary antibody $(1: 1000)$ at $37^{\circ} \mathrm{C}$ for $1 \mathrm{~h}$. Thereafter, PVDF membrane was washed three times with TBST. The protein bands were detected by DAB staining, and the band densities quantified using Image $J$ software (National Institutes of Health). For normalization of the loading, $\beta$-actin was used in the western blot assay.

\section{Reverse transcriptase polymerase chain reaction (RT-PCR) assay}

The mRNA expressions of MCP-1 and CTGF were analyzed by using RT-PCR determination. Total RNA was extracted from the renal cortical tissue by using TRIzol reagent. First strand cDNA were synthesized from $3 \mu \mathrm{g}$ of total RNA. Sequences of the primers used in our investigation were as follows: for CTGF (160bp) 5'-AGCTGCCTACCGACTGGAAGA-3' (forward), 5'-CTTCTCCAGCCTGCAGAAGGT-3 (reverse); for MCP-1(191bp) 5'-CCTGCTGCTACTCATT CAC-3'(forward), 5'-TCTCACTTGGTTCTGGTC3' (reverse); for $\beta$-actin(650bp) 5'CTCTGGTCGTACCACTGGCATTG-3' (forward), 5'-CCTGCTTGCTGATCCACATCTGC-3' (reverse).

PCR experiment was performed under the following selected conditions: first predenaturation at $94{ }^{\circ} \mathrm{C}$ for 3 min followed by 30 cycles of reactions of denaturation at $95{ }^{\circ} \mathrm{C}$ for $50 \mathrm{~s}$, annealing at $61.6{ }^{\circ} \mathrm{C}$ (CTGF), $56.2{ }^{\circ} \mathrm{C}$ (MCP-1), $63.6{ }^{\circ} \mathrm{C}$ ( $\beta$-actin) for $50 \mathrm{~s}$, extension at $72{ }^{\circ} \mathrm{C}$ for $1 \mathrm{~min}$ and last extension for $10 \mathrm{~min}$ at
$72{ }^{\circ} \mathrm{C}$. After amplification, the PCR products were evaluated by $1.5 \%$ agarose gel electrophoresis. Bands were visualized and photographed on a UV trans-illuminator, and $\beta$ actin was used as internal control.

\section{Statistical analysis}

Data were statistically analyzed by using SPSS 16.0. Differences among the groups were analyzed by one-way analysis of variance (ANOVA). The t-test was used between each two groups. Data are presented as mean \pm standard deviation (SD), and $p<0.05$ was regarded as statistically significant.

\section{RESULTS}

\section{Effect of TG on fasting BG and urine biochemical parameters}

After the establishment of DN rat model, the DN rats appeared polydipsia, polyuria and emaciation. In the study, one rat in the TG1 group $(5 \mathrm{mg} / \mathrm{kg}$ ) died. The body weights were significantly decreased in DN group, compared with the NC group. Before treatment with TG, the $B G$ levels of the $D N$ rats were increased by approximately 6-fold, compared with NC rats. After 8 weeks' treatment, the BG levels of diabetic rats were decreased but still higher than the rats in NC group. However, treating these diabetic rats with $T G$ at doses of 5 and 10 $\mathrm{mg} / \mathrm{kg} / \mathrm{d}$ decreased the BG levels, compared with the DN group. The UTP levels of the rats were increased significantly after induction of $\mathrm{DN}$, and treating these DN rats with TG decreased the UTP levels to some extent, compared with the DN group $(p<0.01)$. There was no statistically significant difference between each group with respect to SCr levels (Table 1 ). The BUN levels of the DN group was higher than the NC group ( $p$ $<0.01$ ), and decreased significantly after treatment with TG $(p<0.01)$. The ALB level of TG2 group was higher than the NC group $(p<$ $0.01)$.

\section{Effect of TG on kidney pathology}

The effect of TG on the pathology of kidney was evaluated by H\&E staining and EM analysis. Kidney of the rats in negative control group (NC group) stained with H\&E showed normal renal histological structure of renal parenchyma and glomeruli (Figure 1A). 
Table 1: Effect of TG treatment on fasting BG level and urine biochemical parameters of DN rats

\begin{tabular}{|c|c|c|c|c|c|c|c|c|}
\hline \multirow{2}{*}{ Parameter } & \multicolumn{4}{|c|}{ Statistics (Before intervention ) } & \multicolumn{4}{|c|}{ Statistics (After intervention) } \\
\hline & NC & DN & TG1 & TG2 & NC & DN & TG1 & TG2 \\
\hline Number (n) & 8 & 8 & 7 & 8 & 8 & 8 & 7 & 8 \\
\hline Body weight $(\mathrm{kg})$ & $0.43 \pm 0.03$ & $0.41 \pm 0.02$ & $0.39 \pm 0.03$ & $0.39 \pm 0.06$ & $0.57 \pm 0.08$ & $0.26 \pm 0.02 \ldots \#$ & $0.39 \pm 0.06$ & $0.34 \pm 0.08^{* \# \#}$ \\
\hline $\mathrm{BG}(\mathrm{mmol} / \mathrm{L})$ & $5.24 \pm 1.05$ & $30.99 \pm 2.56$ & $26.50 \pm 4.49^{\prime}$ & $28.34 \pm 4.07^{n}$ & $5.21 \pm 0.58$ & 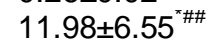 & $18.91 \pm 8.12$ & 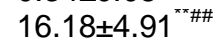 \\
\hline UTP (mg/24h) & $12.09 \pm 1.75$ & $35.31 \pm 3.14$ & $35.80 \pm 3.88^{\pi \pi}$ & $35.89 \pm 5.29^{\pi \pi}$ & $11.68 \pm 1.37$ & $37.19 \pm 5.59^{\pi \times}$ & $21.87 \pm 6.92^{\star \#} \Delta \Delta$ & $18.91 \pm 5.80^{\# \# \Delta} \Delta$ \\
\hline $\mathrm{SCr}(\mu \mathrm{mol} / \mathrm{L})$ & $39.50 \pm 5.29$ & $39.13 \pm 4.99$ & $40.43 \pm 4.43$ & $39.00 \pm 4.66$ & $39.90 \pm 5.60$ & $49.10 \pm 19.10$ & $37.30 \pm 16.20$ & $51.50 \pm 8.50$ \\
\hline $\mathrm{BUN}(\mathrm{mmol} / \mathrm{L})$ & $5.34 \pm 0.45$ & $5.39 \pm 0.44$ & $5.12 \pm 0.33$ & $4.99 \pm 0.41$ & $5.34 \pm 0.36$ & $17.84 \pm 8.71$ & $9.88 \pm 3.69 \Delta \Delta$ & $10.05 \pm 2.29 \Delta \Delta$ \\
\hline $\operatorname{ALB}(\mathrm{g} / \mathrm{L})$ & $23.44 \pm 3.72$ & $23.24 \pm 3.74$ & $24.09 \pm 3.67$ & $24.21 \pm 3.64$ & $23.85 \pm 4.02$ & $23.13 \pm 4.41$ & $28.90 \pm 1.81 \Delta \Delta$ & $31.49 \pm 8.37^{\wedge \prime} \Delta \Delta$ \\
\hline
\end{tabular}

Data are represented as mean $\pm \mathrm{SD}$; compared with the NC group, $p<0.05, " p<0.01$; compared with the DN group, $\Delta p<0.05, \Delta \Delta p<0.01$; compared with before/after

intervention, ${ }^{\#} p<0.05,{ }^{\# \#} p<0.01$

Table 2: Effect of TG treatment on the expression of CTGF and MCP-1, and number of ED-1 positive cells in DN rats

\begin{tabular}{|c|c|c|c|c|}
\hline Parameters & NC & DN & TG1 & TG2 \\
\hline CTGF positive area & $0.1059 \pm 0.0131^{\# \#}$ & $0.4613 \pm 0.1574$ & $0.2302 \pm 0.0214$ & $0.1354 \pm 0.0238^{\# \#}$ \\
\hline MCP-1 positive area & $0.1143 \pm 0.0117^{\# \#}$ & $0.4453 \pm 0.1519^{\pi \pi}$ & 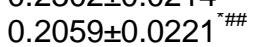 & $0.1749 \pm 0.0506^{\# \#}$ \\
\hline CTGF protein & $0.139 \pm 0.011$ & $0.334 \pm 0.038^{\pi \pi}$ & $0.234 \pm 0.022^{\approx \ldots} \#$ & $0.203 \pm 0.011^{\pi \# \#}$ \\
\hline MCP-1 protein & $0.189 \pm 0.002$ & $0.501 \pm 0.058$ & 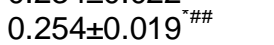 & 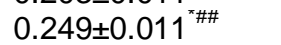 \\
\hline CTGF MRNA & $0.2732 \pm 0.0234^{\# \#}$ & $0.5989 \pm 0.0302$ & $0.4196 \pm 0.0267^{\sim \cdots \#}$ & $0.2904 \pm 0.0651^{\# \# \Delta \Delta}$ \\
\hline MCP-1 mRNA & $0.2421 \pm 0.0219^{\# \#}$ & $0.4335 \pm 0.0240$ & $0.3369 \pm 0.0324$ & $0.2636 \pm 0.0220^{\# \# \Delta \Delta}$ \\
\hline The number of ED-1 positive cells (Glomeruli) & $0.86 \pm 0.61$ & $2.73 \pm 1.02^{\pi x}$ & $1.67 \pm 0.74^{\pi \approx \# \#}$ & $1.41 \pm 0.71^{\pi \times \# \#_{\Delta}}$ \\
\hline The number of ED- 1 positive cells (Glomerular interstitial) & $2.14 \pm 1.08$ & $13.18 \pm 2.19^{\prime \prime}$ & $9.86 \pm 1.63^{\wedge} \# \#$ & $9.49 \pm 1.24^{\wedge \# \#}$ \\
\hline
\end{tabular}

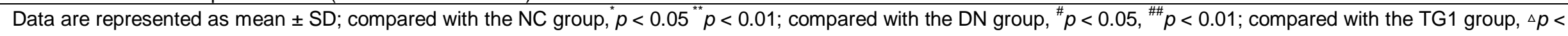
$0.05, \Delta \Delta p<0.01$ 
Figure 1B showed kidney of rat from the positive control group (DN group) with slightly increased glomeruli, thickened glomerular basement membrane, widening mesangial, and vacuolated epithelial lining renal tubules. To some extent, treating DN rats with $5 \mathrm{mg} / \mathrm{kg} / \mathrm{d}$ of TG in TG1 group (Figure $1 \mathrm{C}$ ) and a higher dose of TG (10 $\mathrm{mg} / \mathrm{kg} / \mathrm{d}$ ) in TG2 group (Figure 1D) restored the above-mentioned histopathological changes.
Kidney of the rats in NC group observed under TEM showed normal renal histological structures (Figure 2A). Figure 2B showed kidney of rat from the DN group with thickened glomerular basement membrane, effacement of podocyte foot processes and reduction of the foot processes hiatus number. In Figure $2 \mathrm{C}$ and $2 \mathrm{D}$, kidney tissue of DN rats in TG1 and TG2 treated with different doses of TG (5 and $10 \mathrm{mg} / \mathrm{kg} / \mathrm{d}$ ) reduced these renal damages.

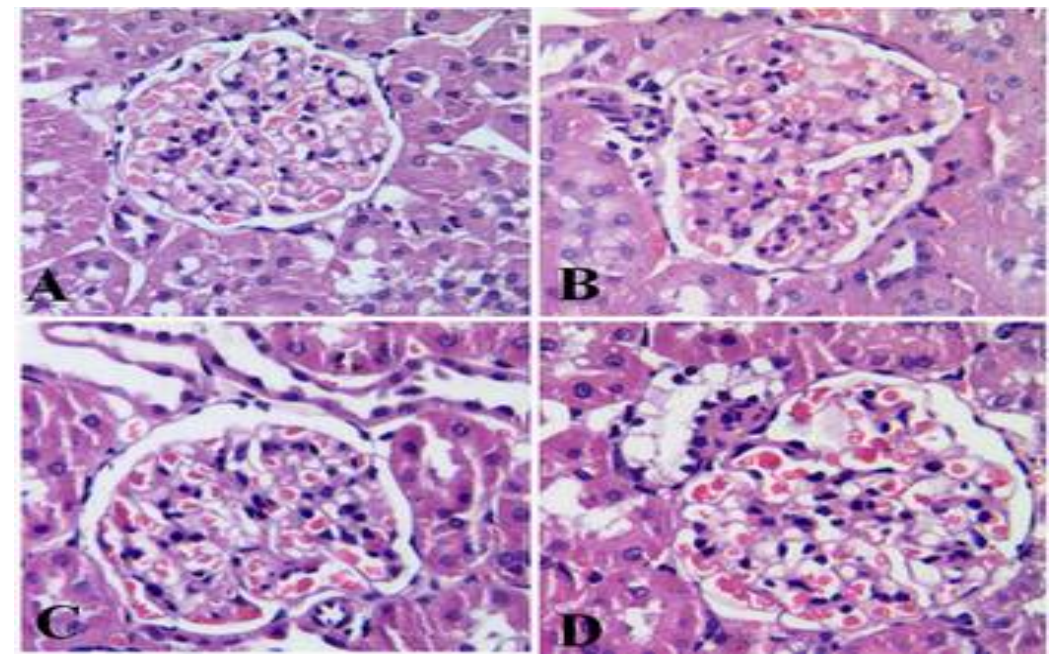

Figure 1: H\&E staining of Kidney in the different groups (400 $\times$ magnification); (A) NC group: Kidney of the rats showed normal renal histological structure of renal parenchyma and glomeruli; (B) DN group: Kidney of rat from the positive control group with slightly increased glomeruli, thickened glomerular basement membrane, widening mesangial and vacuolated epithelial lining renal tubules; (C) TG1 group: Treating DN rats with TG (5 mg/kg/d) reduced the above-mentioned histopathological changes; (D) TG2 group: Treating DN rats with a higher dose of $\mathrm{TG}(10 \mathrm{mg} / \mathrm{kg} / \mathrm{d})$ restored the above-mentioned histopathological changes to some extent
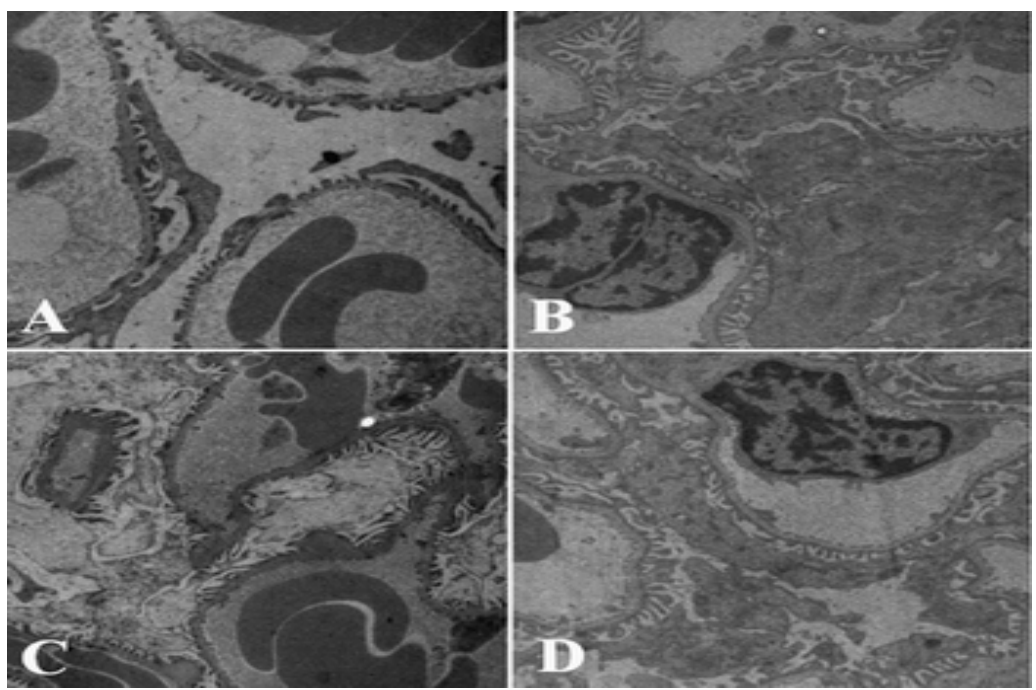

Figure 2: Transmission electron micrographs of kidney in the different groups (7000× magnification); (A) NC group: Kidney of the rats showed normal renal histological structures; (B) DN group: Kidney of rat from the positive control group with thickened glomerular basement membrane, effacement of podocyte foot processes and reduction of the foot processes hiatus number; (C) TG1 group: Treating DN rats with TG (5 mg/kg/d) reduced the above-mentioned renal damage; (D) TG2 group: Treating DN rats with a higher dose of TG (10 mg/kg/d) restored the above-mentioned renal damages to some extent. 


\section{Effect of TG on expressions of MCP-1 and CTGF in kidney tissues}

To investigate the effect of TG on expression of MCP-1 and CTGF in DN rats, immunohistochemistry, western blot and PCR analysis were performed. Immunohistochemical staining of MCP-1 and CTGF in kidney tissues were mainly localized to the glomeruli and tubulointerstitial revealing a significant increase in the DN rats, compared with the NC rats. The increase of MCP-1 and CTGF expression in the kidney of the $D N$ rats was attenuated after treatment with TG (Figure 3 and 4).

Quantitative analysis of these images showed that the expressions of MCP-1 and CTGF in the rats of DN group were significantly increased ( $p$ $<0.01$ ), compared with the NC group, and the expressions of MCP-1 and CTGF in the rats of TG1 and TG2 group were significantly decreased $(p<0.01$ ), compared with the DN group (Table 2). The result of western blot analysis was similar with the immunohistochemistry result. We also quantified MCP-1 and CTGF mRNA expression in kidney tissues of all the groups (Figure 6). RTPCR analysis showed that the expressions of MCP-1 and CTGF mRNA in the kidney of rats of the $\mathrm{DN}$ group were also significantly increased $(p$ $<0.01$ ), compared with the normal control rats. TG significantly reduced these DN-induced increases in the expressions of MCP-1 and CTGF $(p<0.01)$ (Figure 5). This result demonstrated that TG prevents the expressions of MCP-1 and CTGF in rats with diabetic nephropathy.

\section{Effect of TG on infiltration of macrophages}

To evaluate the infiltration of macrophages in the different groups, immunohistochemical staining for the macrophage marker ED-1 was performed. As shown in Figure 7 and Table 2, the number of ED-1 positive cells increased significantly in the kidney of DN group $(p<0.01)$, compared with the NC group. After treatment with TG, the number of ED-1 positive cells in TG1 and TG2 group were decreased significantly $(p<0.01)$, particularly in the TG2 group, compared with the DN group.

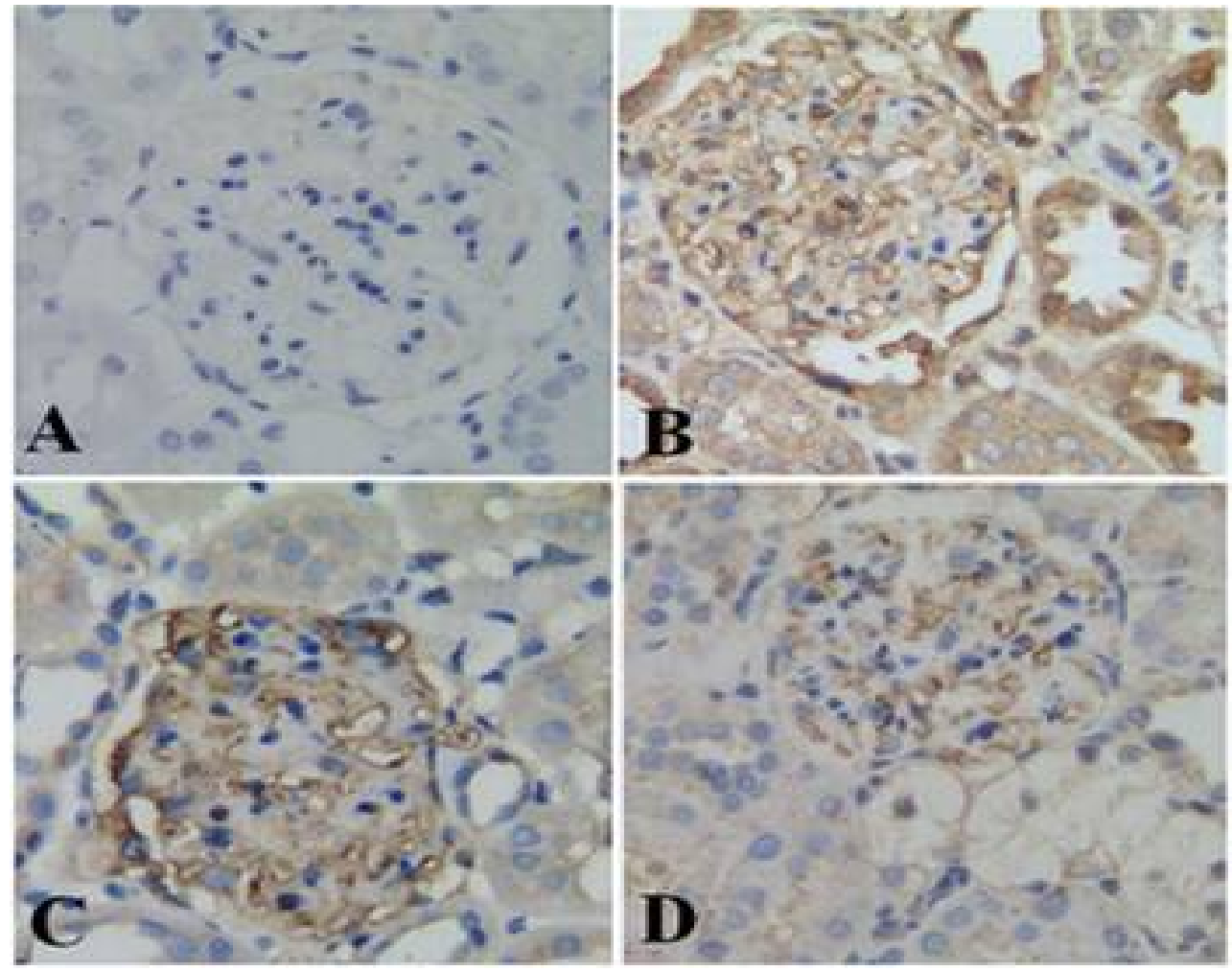

Figure 3: Immunohistochemical staining of MCP-1 in the kidney of different groups (400x magnification); (A) NC group: rat kidneynshowed no brown immunopositive deposits; (B) DN group: Brown immunopositive deposits were largely localized to the glomeruli and tubulointerstitial; (C) TG1 group: Treating DN rats with TG (5 mg/kg/d) reduced the immunopositive deposits; (D) TG2 group: Treating DN rats with TG $(10 \mathrm{mg} / \mathrm{kg} / \mathrm{d})$ reduced the immunopositive deposits 


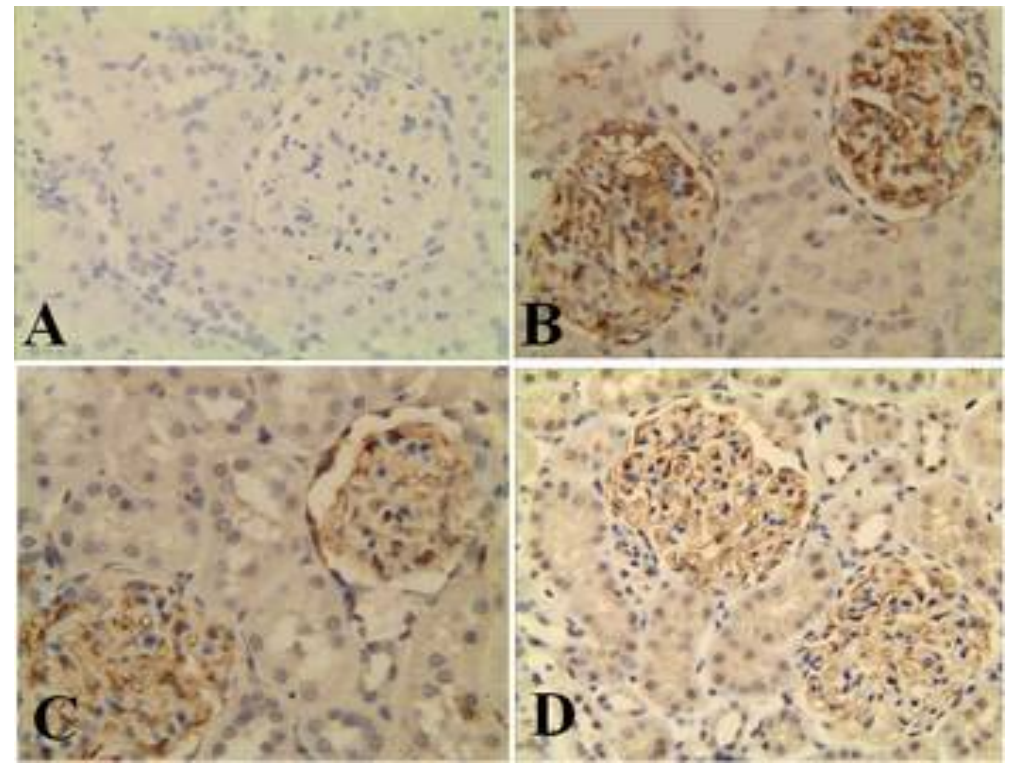

Figure 4: Immunohistochemical staining of CTGF in the kidney of different groups (400x magnification). (A) NC group: Kidney of the rats showed no brown immunopositive deposits; (B) DN group: Brown immunopositive deposits were largely localized to the glomerular and tubulointerstitial; (C) TG1 group: Treating DN rats with TG (5 mg/kg/d) reduced the immunopositive deposits; (D) TG2 group: Treating DN rats with TG (10 mg/kg/d) reduced the immunopositive deposits

\section{NC group DN group TG1 group TG2 group}

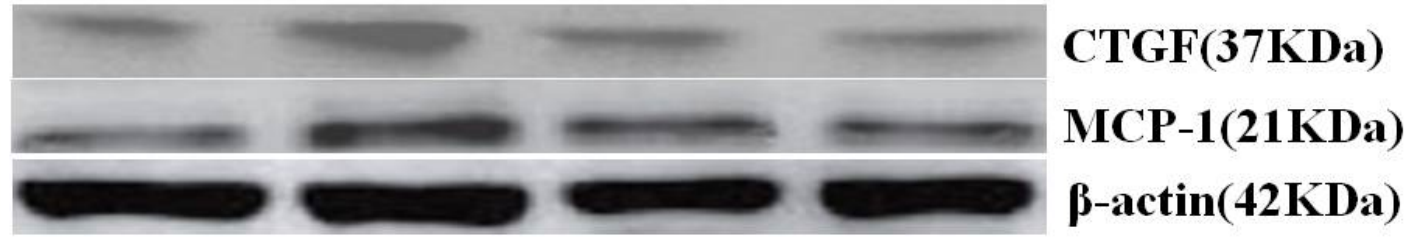

Figure 5: Western blots for CTGF and MCP-1 in the kidney

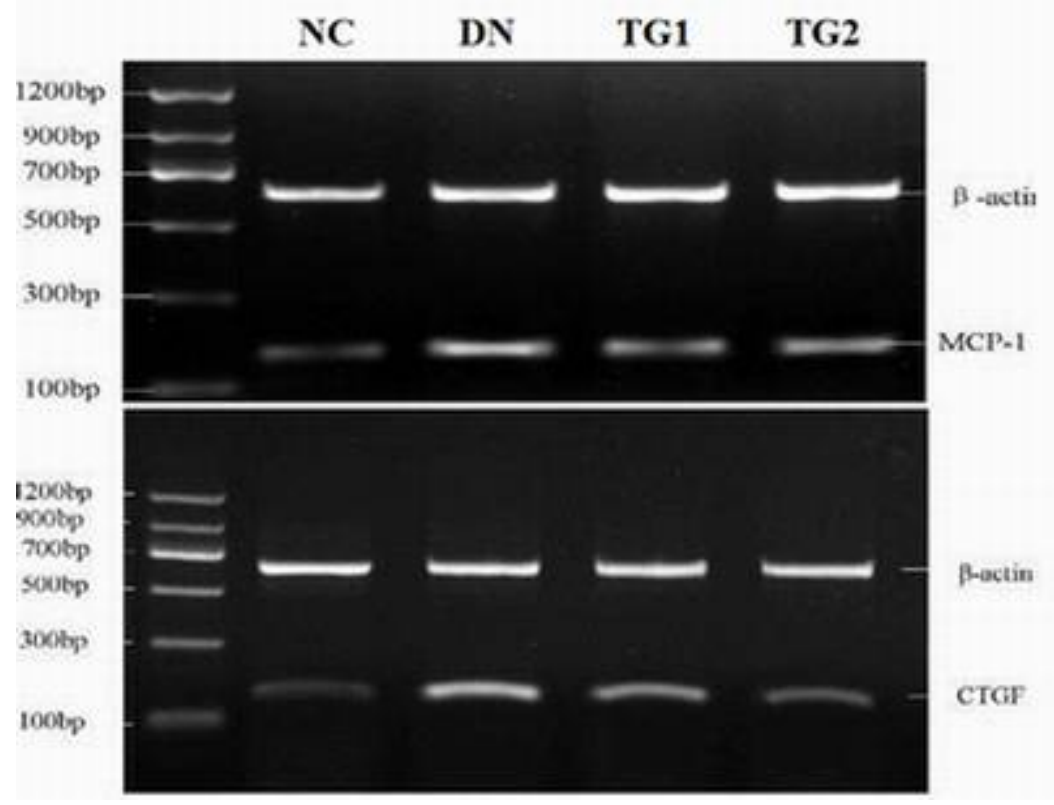

Figure 6: Effect of TG treatment on the expressions of CTGF and MCP-1 mRNA in the kidney. Real-time PCR analysis of the CTGF and MCP-1 genes expression in rats of NC, DN and treated with TG groups (TG1 and TG2 group) 


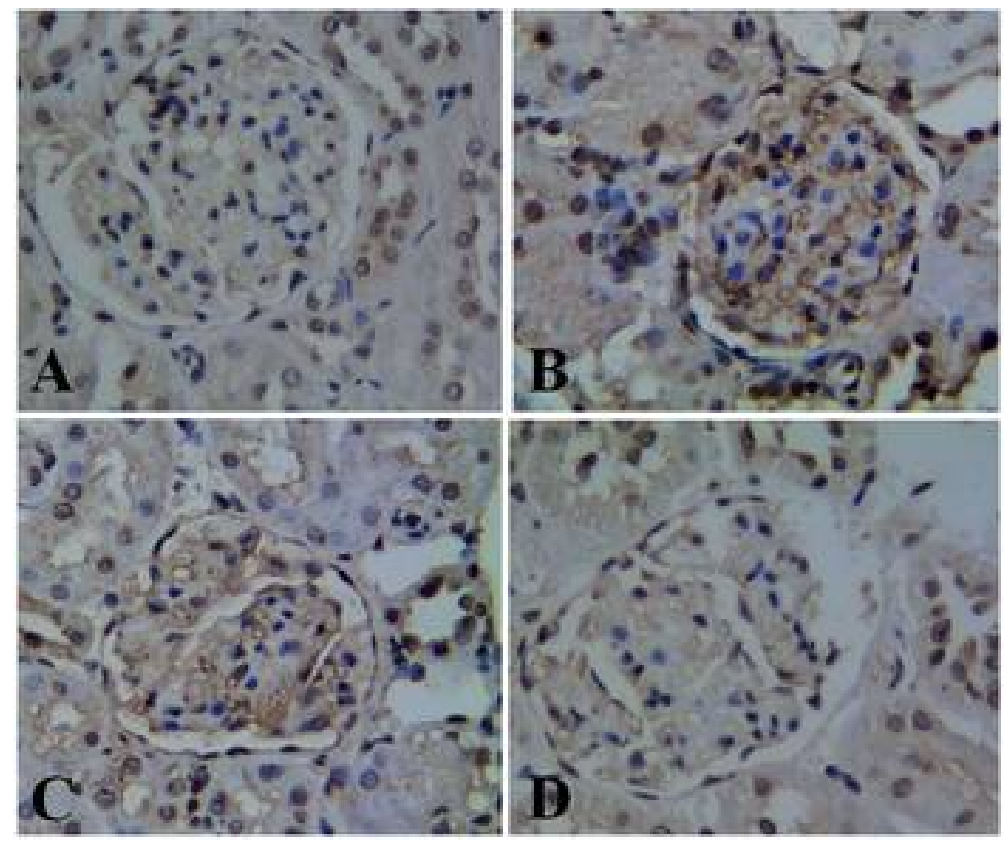

Figure 7: Immunohistochemical staining of ED-1 in the kidney of different groups (400x magnification); (A) NC group: Kidney of the rats showed small amount of brown immunopositive deposits; (B) DN group: Brown immunopositive deposits were largely localized to the glomerular and tubulointerstitial; (C) TG1 group: Treating DN rats with TG $(5 \mathrm{mg} / \mathrm{kg} / \mathrm{d})$ reduced the immunopositive deposits; (D) TG2 group: Treating DN rats with TG (10 $\mathrm{mg} / \mathrm{kg} / \mathrm{d}$ ) reduced the immunopositive deposits

\section{DISCUSSION}

In the last decade, researchers have demonstrated that inflammation and alterations in the immune system play important roles in the occurrence and development of DN [1]. MCP-1 is one of the important inflammatory chemokines for promoting interstitial inflammation, and is found to correlate with severity of the disease in a variety of renal diseases [4].

Infiltration of mononuclear macrophages can stimulate innate renal cell activation and proliferation, which can promote the progression of kidney damage, increase the synthesis and aggregation of type I, III and IV collagen, induce the expression of adhesion molecules, increase the release of lysosomal enzymes and TGF, increase the expression of ECM protein and promote the development of glomerular sclerosis and interstitial fibrosis.

CTGF is an immediate early gene belonging to the CCN protein family, and is highly expressed in the kidney. It's demonstrated that CTGF play a key role in DN caused by renal interstitial fibrosis and glomerulosclerosis, and could be a major cause of kidney damage in diabetes [5]. Urinary level of MCP-1 was observed to be significantly increased and was found to correlate with the degree of renal interstitial fibrosis and tubular atrophy [6]. Additionally, it was confirmed that urinary CTGF level could act as a sensitive early indicator of renal injury. The fact that the concentration of urinary CTGF was positively correlated with the severity of disease makes it helpful to detect, diagnose, and monitor the progression of DN $[7,8]$.

Many previous studies have shown that glomerulosclerosis is associated with macrophage infiltration in $\mathrm{DN}$, and the expressions of MCP-1 and CTGF in mesangial cell were involved in the development of DN [7,9], suggesting that MCP-1 and CTGF play important roles in the pathogenesis of this disease. Thus, MCP-1 and CTGF are potential targets for treating $\mathrm{DN}$, and inhibiting the expressions of MCP-1 and CTGF might be beneficial for treating DN. Our results also demonstrated that expressions of MCP-1 and CTGF were related to the early renal damages of DN rats, and macrophages secreted many profibrotic molecules after entering into the glomeruli and tubulointerstitial area under the chemotactic effect of MCP-1, which in turn promote renal interstitial fibrosis.

These results are consistent with the previous results of Wada [10]. In this study, we found that TG treatment could down-regulate the expressions of MCP-1 and CTGF in kidney and inhibit the macrophage infiltration. In addition, TG can suppress immune response, inhibit the 
proliferation of mesangial cells and matrix, and delay the progression of glomerulosclerosis in DN. Furthermore, TG can alleviate the renal tubular damage via inhibiting the releases of inflammatory mediators and cytokines in renal tubular epithelial cells (TECs). Thus, TG possesses promising protective effect on glomerular podocytes and tubular epithelial cells. Previous investigations reported that extracts of Tripterygium wilfordii Hook $f$ (i.e. triptolide) could suppress the increase of MCP-1 in renal tubular epithelial cells caused by multiple stimuli [11]. The protective effect of TG on the kidneys of diabetic rats may be related to the reduction of CTGF in kidney [12]. Clinical studies also found that TG could significantly reduce proteinuria in $\mathrm{DN}$, delay the onset of disease, and TG was reported to be more effective than the angiotensin receptor blocker (ARBs) drugs [13]. However, the specific mechanisms of TG still need further study.

It has been reported that infiltration of macrophage and abnormal expressions of inflammatory factors are important features of early inflammatory response in $\mathrm{DN}$, and the macrophage is the main target of TG for treating DN [14]. In the present study, we found that expressions of CTGF and MCP-1 in DN rats were increased significantly, compared with that of normal rats. After treatment with TG, the infiltration of macrophage and expressions of CTGF and MCP-1 in kidney were decreased significantly. All these results suggested that TG could reduce kidney damage through downregulating the expressions of CTGF and MCP-1, and decreasing the infiltration of macrophage.

We have further revealed that TG could inhibit the abnormal expressions of CTGF and MCP-1 in the early stages of DN rats, suggesting that TG might reduce glomerular injury through inhibiting aggregation and activated antiinflammatory effects of macrophage. High-dose (10 mg/kg/d) TG was observed to be more effective than the low-dose TG $(5 \mathrm{mg} / \mathrm{kg} / \mathrm{d})$ in down-regulating the expressions of CTGF and MCP-1, especially the CTGF. Furthermore, expressions of Scr and BUN decreased after TG treatment, suggesting that TG may improve the nutritional status of $D N$ rats. The $B G$ of rats increased after induction of DN, by the increase was reversed by treatment with TG. Moreover, high dose of TG $(10 \mathrm{mg} / \mathrm{kg})$ affected the lipid level of the rats by increasing total cholesterol and triglycerides. However, this result is not quite in line with a previous study [15]. Thus further experimental and clinical studies were needed, and it is worth mentioning that dyslipidemia, in addition to the traditional TG adverse effects, should be controlled in the clinical use of TG.

\section{CONCLUSION}

MCP-1 and CTGF play key roles in the occurrence and development of DN. TG exhibits notable protective effect against renal damage via inhibition of the expressions of MCP-1 and CTGF and reduction of macrophage activation. It, therefore, requires further development for treatment of early DN.

\section{CONFLICT OF INTEREST}

The authors declare no conflict of interest associated with this work.

\section{CONTRIBUTION OF AUTHORS}

We declare that this work was done by the authors named in this article and all liabilities pertaining to claims relating to the content of this article will be borne by the authors. Ji-Qiang Zhang, Yan Zhang, Xiao-Li Yin and Wei-Dong Chen conceived, designed and performed the study. Ping Yang, Hai-Feng Zhang, Ya-Ling Guo collected and analyzed the data.

\section{REFERENCES}

1. Mora C, Navarro JF. The role of inflammation as a pathogenic factor in the development of renal disease in diabetes. Curr Diabetes Rep 2005; 5: 399-401.

2. Elmarakby AA, Sullivan JC. Relationship between oxidative stress and inflammatory cytokines in diabetic nephropathy. Cardiovasc Ther 2012; 30: 49-59.

3. Mason RM. Connective tissue growth factor (CCN2), a pathogenic factor in diabetic nephropathy. What does it do? How does it do it? J Cell Commun Signal 2009; 3 : 95-104.

4. Lee EY, Chung $\mathrm{CH}$, Khoury CC, Yeo TK, Pyagay PE, Wang $A$, Chen $S$. The monocyte chemoattractant protein-1/CCR2 loop, inducible by TGF-beta, increases podocyte motility and albumin permeability. $A m \mathrm{~J}$ Physiol-Renal 2009; 297: F85-94.

5. Yokoi H, Mukoyama M, Mori K, Kasahara M, Suganami $T$, Sawai $K$, et al. Overexpression of connective tissue growth factor in podocytes worsens diabetic nephropathy in mice. Kidney Int 2008; 73: 446-455.

6. El Mesallamy HO, Ahmed HH, Bassyouni AA, Ahmed AS. Clinical significance of inflammatory and fibrogenic cytokines in diabetic nephropathy. Clin Biochem 2012; 45: 646-650.

7. Nguyen TQ, Tarnow L, Andersen S, Hovind P, Parving $H H$, Goldschmeding $R$, et al. Urinary connective tissue growth factor excretion correlates with clinical markers of renal disease in a large population of type 1 diabetic 
patients with diabetic nephropathy. Diabetes Care 2006; 29: 83-88

8. Xiao $H Q$, Zhang JE, Ding GH. Determination of connective tissue growth factor in kidney and its value in early diagnosis of diabetic nephropathy. Chin J Diabetes 2006; 14(1): 37-40.

9. Giunti S, Tesch GH, Pinach S, Burt DJ, Cooper ME, Cavallo-Perin $P$, et al. Monocyte chemoattractant protein-1 has prosclerotic effects both in a mouse model of experimental diabetes and in vitro in human mesangial cells. Diabetologia 2008; 51: 198-207.

10. Wada T, Furuichi $K$, Sakai $N$, Iwata $Y$, Yoshimoto $K$, Shimizu $M$, et al. Up-regulation of monocyte chemoattractant protein-1 in tubulointerstitial lesions of human diabetic nephropathy. Kidney Int 2000; 58: 14921499.
11. Li H, Liu ZH, Dai CS, Liu D, Li LS. Triptolide inhibits proinflammatory factor-induced over-expression of class II MHC and B7 molecules in renal tubular epithelial cells. Acta pharmacol Sin 2002; 23: 775-781.

12. $L v H L$, Liu $L Q$. The effect of tripterygium glycosides on the expression of CTGF in diabetic nephropathy. Chin J Integr Tradit Western Nephrol 2009; 10(4): 312-314.

13. Ge YC, Xie HL, Li SJ. Treatment of diabetic nephropathy with Tripterygium wilfordii Hook F extract: a prospective, randomized, controlled clinical trial. Chin J Nephrol Dialysis Transplantation 2010; 19(6): 501-507.

14. Tuttle KR. Linking metabolism and immunology: diabetic nephropathy is an inflammatory disease. J Am Soc Nephrol 2005; 16: 1537-1538.

15. Dong XG: The effect of tripterygium glycosides on lipids in patients with diabetic nephropathy. J Qiqihar Univ Med 2006; 27(4): 416-417. 